\title{
NOTE \\ Primary malignant peripheral nerve sheath tumors arising from the spinal canal invading the abdominal cavity in a dog
}

\author{
Momoko NARITA ${ }^{1)}$, Hidetaka NISHIDA ${ }^{1,3) *}$, Sho GOTO2), Mami MURAKAMI ${ }^{1,2)}$, \\ Hiroki SAKAI ${ }^{1)}$, Kohei NAKATA ${ }^{1,2)}$, Sadatoshi MAEDA ${ }^{1,2)}$ and \\ Hiroaki KAMISHINA ${ }^{1,2)}$ \\ 1) Joint Department of Veterinary Medicine, Faculty of Applied Biological Sciences, Gifu University, \\ 1-1 Yanagido, Gifu, Gifu 501-1193, Japan \\ ${ }^{2)}$ Animal Medical Center, Gifu University, 1-1 Yanagido, Gifu, Gifu 501-1193, Japan \\ ${ }^{3)}$ Department of Advanced Clinical Sciences, Graduate School of Life and Environmental Sciences, \\ Osaka Prefecture University, 1-58 Rinku Ourai Kita, Izumisano, Osaka 598-8531, Japan
}

J. Vet. Med. Sci.

82(4): 452-456, 2020

doi: $10.1292 /$ jvms.19-0113

Received: 27 February 2019 Accepted: 3 February 2020 Advanced Epub:

19 February 2020
ABSTRACT. A 9-year-old neutered male Wire Fox Terrier presented with an 1-month history of hindlimb paresis. Magnetic resonance imaging revealed a contrast-enhanced mass at the level of the L2 vertebral canal. The dog became paraplegic with no deep perception of the hindlimbs, and the mass was surgically removed. The histopathological diagnosis was of a malignant peripheral nerve sheath tumor (MPNST). The dog suffered a relapse of right hindlimb ataxia at 225 days after the surgery. The dog died 434 days after the surgery. Necropsy found a large mass in the abdominal cavity invading from the L2-nerve. This is the first report of MPNST invading the abdominal cavity through the nerve root.

KEY WORDS: dog, invasion, peripheral nerve sheath tumor

Peripheral nerve sheath tumors (PNSTs) arise from endoneurial fibroblasts and/or schwann cells that surround the axons of neurons in peripheral nerves. Malignant PNSTs (MPNSTs) preferentially invade the fascicles of the nerve [2, 17]. This invasiveness explains the higher recurrence rate after surgical resection [6]. In dogs, MPNSTs arise most commonly from peripheral nerves, spinal roots, and cranial nerves [11]. PNSTs arising within the abdomen have been observed, including in the spleen, liver, kidney, adrenal gland, greater omentum, and diaphragm in dogs $[1,14]$. Although there are a small number of reports of PNSTs invading into the thoracic cavity through the nerve root in dogs, humans, and cows $[4,9,12,16]$, this is the first report in humans or animals of MPNSTs invading into the abdominal cavity through the nerve root.

A 9-year-old, neutered male Wire Fox Terrier was admitted with a history of hind limb paresis for approximately 1 month. Treatment with steroids gave an initial improvement, but clinical signs deteriorated. On the initial referral to hospital (day 1), all vital parameters were normal. Serum biochemical examinations found a marked elevation of alanine transaminase (ALT, $428 \mathrm{U} / l$ ), alkaline phosphatase (ALP, 3,313 U/l), creatine phosphokinase (CPK, 1,373 IU/l), lipase (824 IU/l), and total bile acids (TBA, $26.1 \mu \mathrm{mol} / l)$. The whole cell blood count (CBC) was in normal range. His mental status was alert during the neurological examination. Postural reaction deficits of the hind limbs were noted, with paraparesis and urinary incontinency. Spinal reflexes of all limbs and cranial nerve functions were unremarkable. The lesion was suspected to be localized in the T3-L3 spinal cord segment. Radiographs and computed tomography (CT) showed enlargement of the spinal canal at the L2 level. A magnetic resonance imaging (MRI) scan using a 0.4 T unit (APERTO Eterna; Hitachi Healthcare, Kashima, Japan) was performed. The sequences included T2-weighted images (T2WI; TR, 4,500; TE, 13), fluid-attenuated inversion recovery (FLAIR; TR, 9,000; TE, 100), T1-weighted images (T1WI; TR, 350; TE, 13), and postcontrast T1WI. Images of the transverse, dorsal, and sagittal planes were acquired in each sequence. Scrutiny of the images revealed a mass at the level of the L2 vertebral canal, mainly on the right side. The mass appeared isointense on T1WI, and hyperintense on T2WI and FLAIR. This lesion was enhanced by gadoliniumbased contrast medium (0.1 mmol/kg, Omniscan, Daiichi Sankyo, Tokyo, Japan; Fig. 1A, 1B). The mass compressed the spinal cord from the right side.

On day 7, the dog was paraplegic, without deep perception of the pelvic limbs. After a consultation with the owner, surgical removal of the tumor was chosen. The surgery was performed via a right dorsal approach to the L2 vertebra. A right 


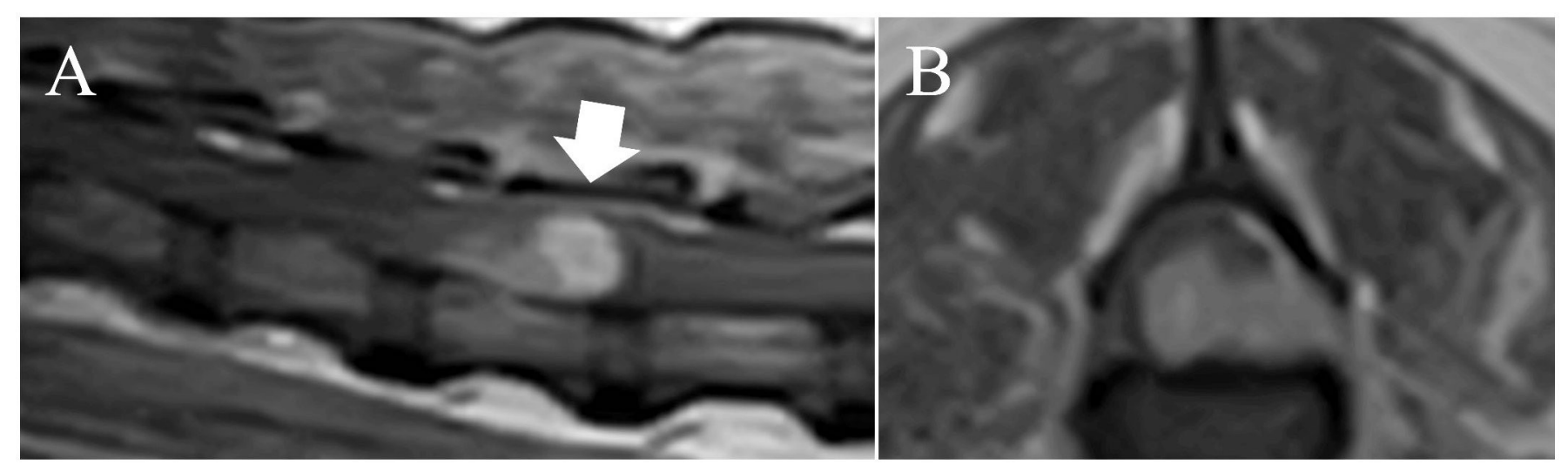

Fig. 1. Sagittal (A) and transverse (B) postcontrast T1WI at initial referral. (A) Contrast-enhanced mass was observed at the level of the L2 vertebral canal (arrow). (B) The mass was mainly on the right side and it compressed the spinal cord.

hemilaminectomy was performed between L1-L3. The mass was found in intradural-extramedullary region and in the nerve root. Gross total resection of the mass of nerve root was performed. However, residual tumor was observed in the peripheral nerve intraoperatively. Tissue samples were fixed in $10 \%$ neutral buffered formalin, processed routinely and embedded in paraffin wax. Sections were cut at $4 \mu \mathrm{m}$ thick and stained with hematoxylin and eosin (HE) and used for immunohistochemistry. Histopathologically, the tumor was consisted with pleomorphic spindle cells, arranged mostly in interwoven with a round or elliptical nucleus. Mitotic figures were occasionally observed (Fig. 2A). Immunohistochemical staining (IHC) was performed using the polymer immunocomplex method (Envision ${ }^{\mathrm{TM}}$ Dakocytomation, Glostrup, Denmark) with 3,3-diaminobenzidine (DAB) and counterstained with Mayer's hematoxylin. The primary antigen to evaluate of MPNST used for IHC were as follows; rabbit polyclonal anti-S100 protein (Dakocytomation), rabbit polyclonal anti-neuron-specific enolase (NSE, Abcam, Cambridge, UK), mouse monoclonal anti-Class III $\beta$-Tubulin (Promega, Madison, WI, USA), mouse monoclonal anti-nerve growth factor receptor (NGFR, Leica Biosystems Newcastle Ltd., Newcastle, UK), rabbit polyclonal anti-nestin (IBL, Gunma, Japan), rabbit polyclonal anti-glial fibrillary acidic protein (GFAP, Dako Japan, Tokyo, Japan), and rabbit polyclonal anti- $\alpha$-smooth muscle actin ( $\alpha$-SMA, Dakocytmation). Antigen retrieval pre-treatment was carried out by heating the tissue sections in target retrieval solutions ( $\mathrm{pH} 6.0$ or high $\mathrm{pH}$, Dako) in an autoclave for either $15 \mathrm{~min}$ or $1 \mathrm{~min}$ at $121^{\circ} \mathrm{C}$. To quench endogenous peroxidase activity, the specimens were incubated with $0.3 \%$ hydrogen peroxide in methanol for $20 \mathrm{~min}$ at room temperature. They were then treated with Protein Block Serum-free solution (Dako) for $30 \mathrm{~min}$ to prevent the binding of nonspecific proteins to primary antibodies. The primary antibodies were incubated overnight at $4{ }^{\circ} \mathrm{C}$. Sections were rinsed after every step with phosphate-buffered saline (PBS) containing 0.1\% Tween 20 (Sigma-Aldrich, St. Louis, MO, USA). For the negative control, the primary antibodies were replaced with PBS. The neoplastic cells were positive for S-100, NSE, NGFR, Class III $\beta$-tubulin, nestin, GFAP and negative for $\alpha$-SMA (Fig. 2B-H). Based on histological features and immunohistochemical expression, this tumor was diagnosed as MPNST classified as of the root group. On day 12 the dog was ambulatory, and controlled urination by himself. With the consent of the owner, the postoperative radiotherapy of the L2 region was initiated on day 26 (2 Gy daily, total 42 Gy) until day 57.

Hind limb ataxia was observed on day 232, and neurological examination detected reduction of postural reactions in the hind limbs. MRI revealed an intramedullary lesion at the level of L1-L2, which was hyperintense on a T2W/FLAIR image and mild contrast enhancement of the lesion. Treatment with prednisolone initially yielded improvement, but the dog was paraplegic without pain perception of the pelvic limbs. On day 431, anorexia, weight loss, and abdominal distension was observed in the clinic. $\mathrm{CBC}$ and serum biochemical examinations revealed anemia [hematocrit (HCT), 21.1\%], Leukocytosis [white blood cells (WBC), 54,000 cells $/ \mu l$, and marked elevation of blood urea nitrogen (BUN, $62 \mathrm{mg} / \mathrm{d} l$ ). The mass was detected in the abdominal cavity, and the owner did not request further treatment. On day 441, the dog died.

On necropsy, a large mass $(22 \times 19 \times 17 \mathrm{~cm})$ was occupying in the abdominal cavity (Fig. 3A). Peritonitis, intestinal necrosis, and intestinal perforation were also observed. The abdominal mass had invaded from the right side of the L2-nerve (Fig. 3B). Nodules were noted on the greater omentum and the intraperitoneal surface of the diaphragm. The histopathological examination revealed recurrence of MPNST in the spinal cord. The tumor was consisted with pleomorphic spindle cells, arranged mostly in interwoven with a round or elliptical nucleus. Mitotic figures were occasionally observed. Similar histopathological features of the tumor cells were also observed in the abdominal mass, and the nodules on the greater omentum and the intraperitoneal surface of the diaphragm. Immunohistochemistry for Ki-67 was performed as described previously [8]. The percentage of Ki-67-positive cells in each tumor tissue, indicating as a proliferative index, was calculated from the proportion of reddish brown-positive nuclei in a total of over 1,000 neoplastic cells (from more than 5 high-power fields) (Fig. 4A, 4B). The index of intradural-extramedullary masses at first surgery and necropsy were $34.6 \%$ and $26.5 \%$, respectively.

In dogs, most MPNSTs commonly affect spinal nerve root in the lower cervical region [3]. Median relapse free intervals of the root group after surgery was 1 month (range, 0 to 14 months), therefore most cases were euthanized due to the sign of relapse [3]. In this study, the dog had approximate 7 months of free relapse period, probably because of the radiotherapy [10, 13]. The 

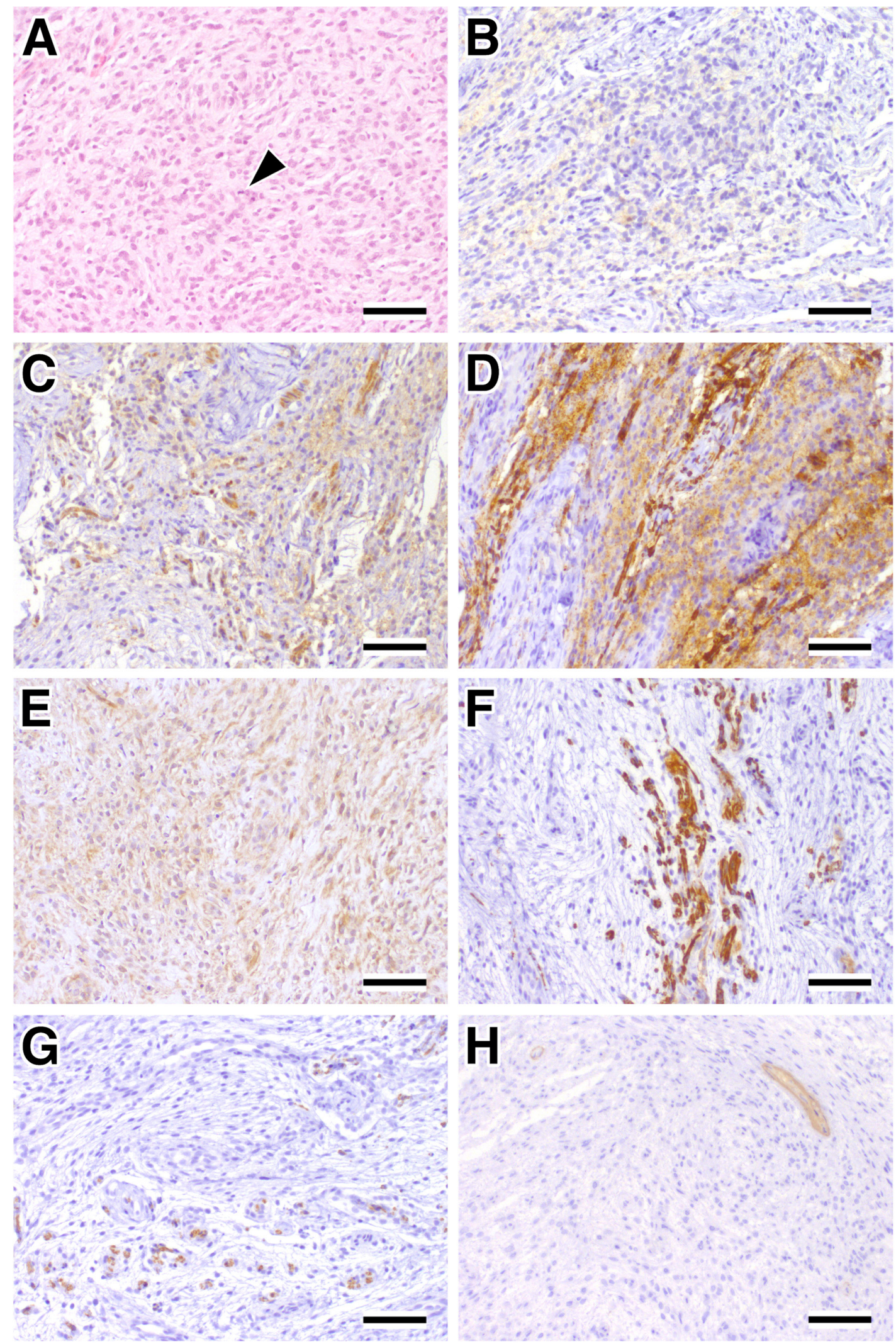

Fig. 2. Histological and immunohistochemical staining of the tumor. (A) The mass consisted of density packed tumor cells and spindle-shaped cells arranged in interwoven bundles. Mitotic figure was occasionally observed (arrow head, mitotic rate: $0.3 \%$ ). Hematoxylin and eosin stain. (B-G) The tumor cells were stained positively for S-100 (B), neuron-specific enolase (C), nerve growth factor receptor (D), nestin (E) and a part of the tumor cells showed positive reaction for Class III $\beta$-Tubulin (F) and glial fibrillary acidic protein $(\mathrm{G})$. As expected, the tumor cells were negative for $\alpha$-smooth muscle actin (H). Bars $=200 \mu \mathrm{m}$. 


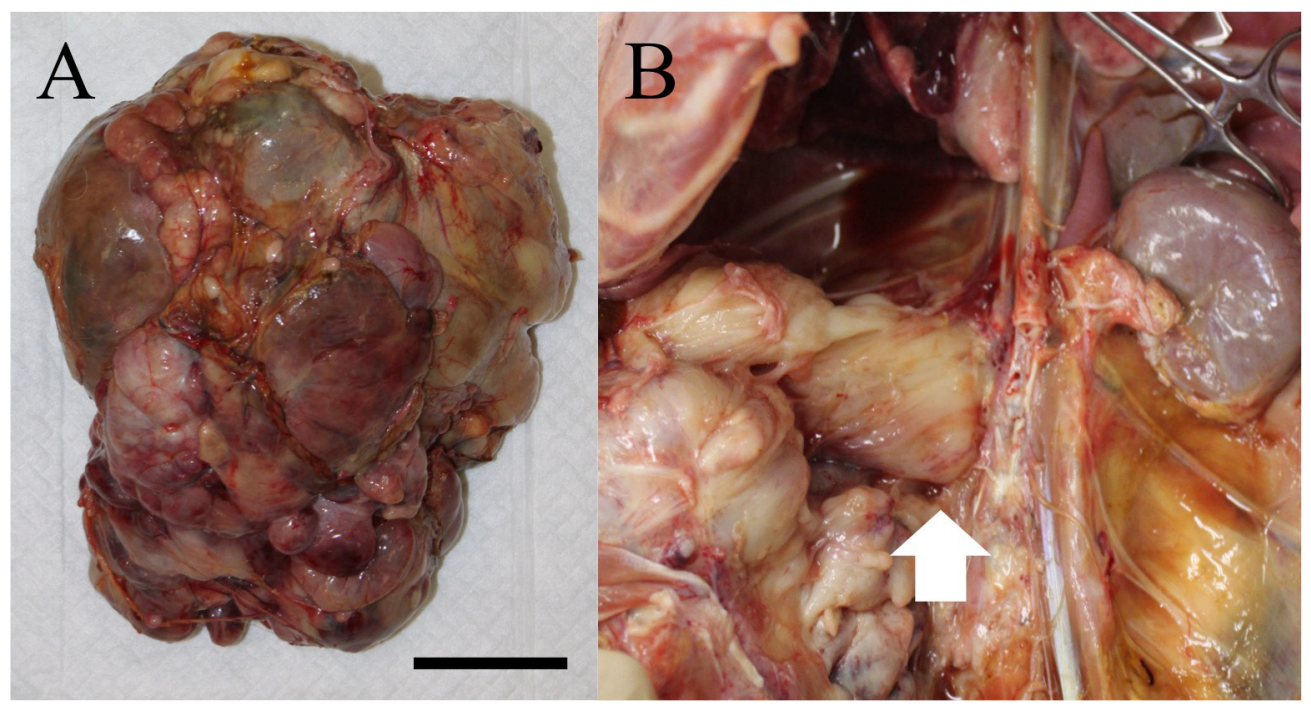

Fig. 3. Gross appearance of the abdominal mass at necropsy. (A) A large mass of dimension $22 \times 19 \times$ $17 \mathrm{~cm}$ occupied the abdominal cavity. The mass had a smooth, dark whitish-red surface. $\mathrm{Bar}=5 \mathrm{~cm}$. (B) The abdominal mass developed from the right side of the L2-nerve (arrow).

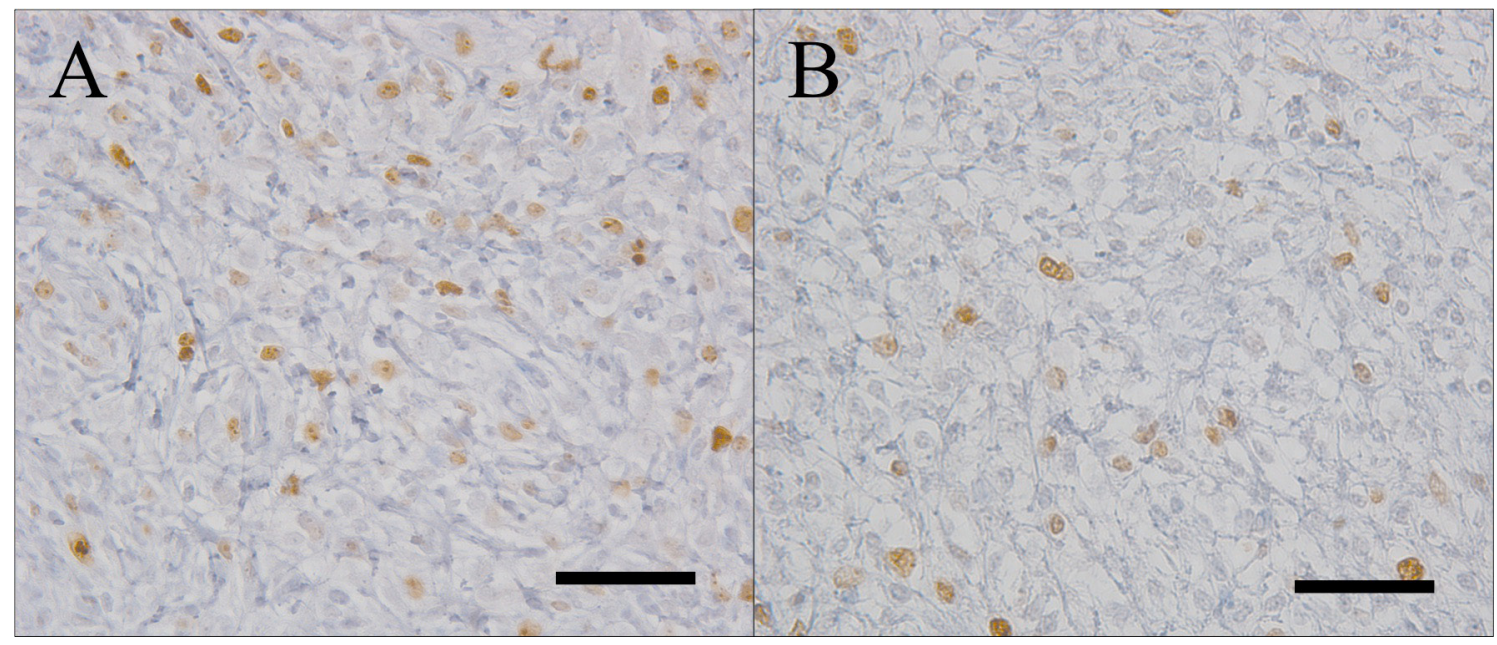

Fig. 4. Immunohistochemical for Ki-67 of the intradural extramedullary mass at surgery (A) and the abdominal mass at necropsy (B). Positive nuclei for Ki-67 were observed. Bars $=50 \mu \mathrm{m}$.

abdominal mass was formed by the invasion into the abdominal cavity along the sympathetic nerve.

To test for high grade or malignant transformation, Ki-67 immunohistochemistry was performed. In human PNSTs, Ki-67 can be used for grading or predicting prognosis [18-20]. The expression of Ki-67 was about $20-30 \%$ at the time of the surgery and necropsy. In humans, the index of Ki-67 was reported to be greater than $5 \%$ in high grade MPNSTs $[19,20]$. The survival time was shorter in human patients having Ki-67 index exceeding $25 \%$. In canine cutaneous PNSTs, the expression of Ki-67 was ranged $0.6-80.9 \%$, showing higher index in MPNSTs $(35.4+27.8 \%)$ than those in PMSTs $(16.3+17 \%)$ [15]. The expression of Ki-67 in primary and metastatic lesion in the case were $34.6 \%$ and $26.5 \%$, respectively, both of which were greater than $25 \%$, implying that the MPNST in the case was highly aggressive. Gracia et al. [5] reported that the Ki-67 was higher in the metastatic lesion than in the primary lesion in dogs [5]. There are some variations, increasing (24\%), stable (66\%), and decreasing (10\%) of Ki-67 in metastatic lesion in human neuroendocrine tumors [7]. Since Ki-67 can be used for grading or predicting prognosis in human PNSTs [18-20] and in canine PNSTs [15], Ki-67 is an available marker for physiological states and prognosis in MPNSTs. To our knowledge, this is the first report in humans or animals on the invasion of MPNSTs into the abdominal cavity from the vertebral canal through the nerve root. 


\section{REFERENCES}

1. Anderson, G. M., Dallaire, A., Miller, L. M. and Miller, C. W. 1999. Peripheral nerve sheath tumor of the diaphragm with osseous differentiation in a one-year-old dog. J. Am. Anim. Hosp. Assoc. 35: 319-322. [Medline] [CrossRef]

2. Angelov, L., Davis, A., O'Sullivan, B., Bell, R. and Guha, A. 1998. Neurogenic sarcomas: experience at the University of Toronto. Neurosurgery 43: 56-64, discussion 64-65. [Medline] [CrossRef]

3. Brehm, D. M., Vite, C. H., Steinberg, H. S., Haviland, J. and van Winkle, T. 1995. A retrospective evaluation of 51 cases of peripheral nerve sheath tumors in the dog. J. Am. Anim. Hosp. Assoc. 31: 349-359. [Medline] [CrossRef]

4. Dezfoulian, O., Jamshidi, S., Ghaffari, M. S., Capucchio, M. T., Masoudifard, M. and Jahanzad, I. 2010. Unusual histopathological findings in a young Pekingese dog with intrathoracic malignant peripheral nerve sheath tumour. Comp. Clin. Pathol. 19: 217-220. [CrossRef]

5. García, P., Sánchez, B., Sánchez, M. A., González, M., Rollán, E. and Flores, J. M. 2004. Epithelioid malignant peripheral nerve sheath tumour in a dog. J. Comp. Pathol. 131: 87-91. [Medline] [CrossRef]

6. Gross, T. L., Ihrke, P. J., Walder, E. J. and Affolter, V. K. 2005. Neural and perineural tumors. pp. 789-796. In: Skin Diseases of the Dog and Cat: Clinical and Histopathologic Diagnosis, 2nd ed. (Gross, T. L., Ihrke, P. J., Walder, E. J. and Affolter, V. J. eds.), Blackwell Publishing, Ames.

7. Keck, K. J., Choi, A., Maxwell, J. E., Li, G., O’Dorisio, T. M., Breheny, P., Bellizzi, A. M. and Howe, J. R. 2017. Increased grade in neuroendocrine tumor metastases negatively impacts survival. Ann. Surg. Oncol. 24: 2206-2212. [Medline] [CrossRef]

8. Kodama, A., Sakai, H., Matsuura, S., Murakami, M., Murai, A., Mori, T., Maruo, K., Kimura, T., Masegi, T. and Yanai, T. 2009. Establishment of canine hemangiosarcoma xenograft models expressing endothelial growth factors, their receptors, and angiogenesis-associated homeobox genes. BMC Cancer 9: 363. [Medline] [CrossRef]

9. Kourea, H. P., Bilsky, M. H., Leung, D. H., Lewis, J. J. and Woodruff, J. M. 1998. Subdiaphragmatic and intrathoracic paraspinal malignant peripheral nerve sheath tumors: a clinicopathologic study of 25 patients and 26 tumors. Cancer 82: 2191-2203. [Medline] [CrossRef]

10. Lacassagne, K., Hearon, K., Berg, J., Séguin, B., Hoyt, L., Byer, B. and Selmic, L. E. 2018. Canine spinal meningiomas and nerve sheath tumours in 34 dogs (2008-2016): Distribution and long-term outcome based upon histopathology and treatment modality. Vet. Comp. Oncol. 16: 344-351. [Medline] [CrossRef]

11. LeCouteur, R. A. 1996. Tumors of the nervous system. pp. 393-419. In: Small Animal Clinical Oncology, 2nd ed. (Withrow, S. J. and MacEwen, E. G. eds.), WB Saunders, Philadelphia.

12. Ramirez, O. 3rd., McDorman, K., Dennis, P. and Hunt, E. 1999. Radiographic diagnosis: multicentric schwannoma in an adult Holstein-Freisian cow. Vet. Radiol. Ultrasound 40: 148-150. [Medline] [CrossRef]

13. Siegel, S., Kornegay, J. N. and Thrall, D. E. 1996. Postoperative osteoperative irradiation of spinal cord tumors in 9 dogs. Vet. Radiol. Ultrasound 37: 150-153. [CrossRef]

14. Suzuki, S., Uchida, K. and Nakayama, H. 2014. The effects of tumor location on diagnostic criteria for canine malignant peripheral nerve sheath tumors (MPNSTs) and the markers for distinction between canine MPNSTs and canine perivascular wall tumors. Vet. Pathol. 51: $722-736$. [Medline] [CrossRef]

15. Teixeira, S., Amorim, I., Rêma, A., Faria, F. and Gärtner, F. 2016. Molecular heterogeneity of canine cutaneous peripheral nerve sheath tumors: a drawback in the diagnosis refinement. In Vivo 30: 819-827. [Medline] [CrossRef]

16. Uchida, K., Nakayama, H., Sasaki, N., Tateyama, S. and Goto, N. 1992. Malignant schwannoma in the spinal root of a dog. J. Vet. Med. Sci. 54: 809-811. [Medline] [CrossRef]

17. Wanebo, J. E., Malik, J. M., VandenBerg, S. R., Wanebo, H. J., Driesen, N. and Persing, J. A. 1993. Malignant peripheral nerve sheath tumors. A clinicopathologic study of 28 cases. Cancer 71: 1247-1253. [Medline] [CrossRef]

18. Watanabe, T., Oda, Y., Tamiya, S., Kinukawa, N., Masuda, K. and Tsuneyoshi, M. 2001. Malignant peripheral nerve sheath tumours: high Ki67 labelling index is the significant prognostic indicator. Histopathology 39: 187-197. [Medline] [CrossRef]

19. Yamaguchi, U., Hasegawa, T., Hirose, T., Chuman, H., Kawai, A., Ito, Y. and Beppu, Y. 2003. Low grade malignant peripheral nerve sheath tumour: varied cytological and histological patterns. J. Clin. Pathol. 56: 826-830. [Medline] [CrossRef]

20. Zhou, H., Coffin, C. M., Perkins, S. L., Tripp, S. R., Liew, M. and Viskochil, D. H. 2003. Malignant peripheral nerve sheath tumor: a comparison of grade, immunophenotype, and cell cycle/growth activation marker expression in sporadic and neurofibromatosis 1-related lesions. Am. J. Surg. Pathol. 27: 1337-1345. [Medline] [CrossRef] 